\title{
Optimal design of micro-mechanisms by the homogenization method
}

\author{
Grégoire Allaire - François Jouve \\ Centre de Mathématiques Appliquées (UMR 7641) \\ École Polytechnique, 91128 Palaiseau \\ Gregoire.Allaire@polytechnique.fr, Francois.Jouve@polytechnique.fr
}

\footnotetext{
ABSTRACT. The design of mechanisms for building micro-tools can be viewed as a shape optimization problem with a peculiar objective function. We propose such an optimization method based on homogenization, which is called topology optimization.

RÉSUMÉ. La conception optimale de mécanismes, destinés par exemple à la fabrication de micro-mécanismes peut-être envisagée comme un problème d'optimisation de formes avec une fonction-coût particulière. Nous présentons ici une relaxation partielle du problème permettant le développement de méthodes numériques efficaces.

KEYWORDS: micro-mechanisms, shape optimization, homogenization.

MOTS-CLÉS : micro-mécanismes, optimisation de formes, homogénéisation.
} 


\section{Introduction}

The use of homogenization techniques in structural topology and shape optimization led to the development of new methods and efficient algorithms (see for example [ALL 00b], [ALL 97], [ALL 93], [BEN 88]). They are able to capture, on a given mesh, an optimal shape without any restrictions on its topology. However, theoretical results are restricted to compliance - for one or more loading cases - or eigenfrequency optimization. In these cases, particular microstructures are known to be optimal, and they even can be characterized in an explicit way for one loading case or the lower eigenfrequency. Of course, many refinements, purely numerical, have been done to handle more general objective functions. They are oftenly based on the use of fictitious materials (for example "power-law" materials [ROZ 95]), or of sub-optimal materials (for example, obtained by homogenization of a perforated periodic cell).

Among all the possible material properties, choosing a particular subclass of composites gives a partial relaxation of the problem. This subclass must be rich enough to allow a good approximation of the optimal composites, yielding good properties for the numerical algorithms (fast convergence, global minima); on the other hand it must be as explicit as possible for a good efficiency.

The subclass of the sequential laminates of any order is a good candidate: their characterization is done through a small number of parameters. Moreover, their macroscopic properties can be explicitly computed as functions of these parameters, and they are optimal in the particular cases of compliance and eigenvalues ([ALL 00b], [ALL 97], [ALL 93]). A partial relaxation of a shape optimization problem using the sequential laminates have been introduced in [ALL 00a]. In this more general case, the theory is not fully established but efficient numerical algorithms can be shown. A gradient method, with a projection step, have been proposed. It involves the resolution of an adjoint problem and the computation of the objective function gradients with respect to shape variables, i.e. parameters that describe the composites in each point of the domain. We present here a similar approach, modified in order to allow the computation of optimal mechanisms (cf. [SIG 97], [SIG 99]). This updated algorithm is useful in designing micro-mechanisms (MEMS), etched on small pieces of Silicium. In this type of structures, traditional junctions (like ball-and-socket joint, articulations, springs) cannot be done. Their potential movement is only due to their shape.

\section{Setting of the problem}

We consider a bounded domain $\Omega \in \mathbb{R}^{N}(N=2,3)$ filled with two linearly elastic materials, characterized by their Hooke's laws $A$ and $B$ and the corresponding Lamé coefficients $\left(\lambda_{A}, \mu_{A}\right)$ and $\left(\lambda_{B}, \mu_{B}\right)$. We suppose that we have the following relations between coefficients: $0<\mu_{B}<\mu_{A}, 0<\lambda_{B}+\frac{2 \mu_{B}}{N}<\lambda_{A}+\frac{2 \mu_{A}}{N}$. In the context of shape optimization of mechanisms, the $B$ material is supposed to "tend" to void, i.e. $\left(\lambda_{B}, \mu_{B}\right) \rightarrow(0,0)$ so that the final shapes are made of only one phase. In the following, the two phases are considered as non-degenerated. A rigorous justification 
of the passage to the limit in the case of optimization of the compliance can be found in [ALL 97]. Note that in numerical computations, the weak phase is never totally degenerated to avoid implementation problems.

Let $\chi \in L^{\infty}(\Omega ;\{0,1\})$ denote the characteristic function of phase $A$. A Hooke's law on the whole domain can be defined by $\Omega$ by $A_{\chi}=\chi A+(1-\chi) B$. The displacement field of the structure is then the unique solution in $H_{0}^{1}(\Omega)^{N}$ of

$$
\left\{\begin{array}{rlll}
-\operatorname{div}\left(A_{\chi} e\left(u_{\chi}\right)\right) & =f & \text { in } \Omega \\
u_{\chi} & =0 & \text { on } \partial \Omega
\end{array}\right.
$$

where $e\left(u_{\chi}\right)=\left(\nabla u+\nabla^{t} u\right) / 2$ denotes the strain tensor and $f \in L^{2}(\Omega)^{N}$ a given volume force. For simplicity, we have chosen to work with a model problem with Dirichlet boundary conditions, but more general surface loadings or boundary conditions are possible. We address the following two-phase optimal design problem:

$$
\inf _{\chi \in L^{\infty}(\Omega ;\{0,1\})} J(\chi)
$$

with an objective function $J$ defined by

$$
J(\chi)=\left(\int_{\Omega} \chi(x) C(x)\left|u(x)-u_{0}(x)\right|^{\alpha} d x\right)^{\frac{1}{\alpha}}+\ell \int_{\Omega} \chi(x) d x,
$$

where $\ell$, a Lagrange multiplier for a volume constraint on phase $A, 1 \leq \alpha<2 N /(N-$ 2), $C \in L^{\infty}(\Omega)$ and $u_{0} \in H^{1}(\Omega)^{N}$ are given. For a given external load, this function amounts to find a structure or mechanism attaining a prescribed displacement $u_{0}$. When $\chi(x) \equiv 1$ is not a trivial solution of (1) with $\ell=0$, which is the case if $u_{0} \neq 0$, the volume constraint can be neglected taking $\ell=0$.

It is known that (1) is ill-posed. After relaxation, it can be shown that there exists generalized solutions to this type of problem (cf. for example [KOH 86], [MUR 85]). These generalized designs are defined as a distribution in the whole domain of composite materials obtained by mixing on a microscopic scale the two phases $A$ and $B$. The composite materials are parametrized by two functions: the density $\theta(x) \in[0,1]$ of phase $A$ and the microstructure or geometric arrangement of the two phases (yielding different effective Hooke's laws $\left.A^{*}(x)\right)$ at each point $x \in \Omega$. By homogenization theory, the relaxed formulation of (1) turns out to be:

$$
\min _{\left(\theta, A^{*}\right) \in \mathcal{C D}} J^{*}\left(\theta, A^{*}\right)
$$

with the generalized objective function

$$
J^{*}\left(\theta, A^{*}\right)=\left(\int_{\Omega} \theta(x) C(x)\left|u(x)-u_{0}(x)\right|^{\alpha} d x\right)^{\frac{1}{\alpha}}+\ell \int_{\Omega} \theta d x,
$$

where $u(x)$ is the unique solution in $H_{0}^{1}(\Omega)^{N}$ of the homogenized problem

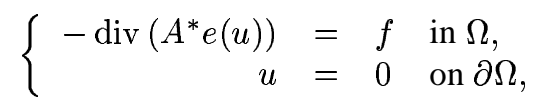


and $\mathcal{C D}$ is the space of generalized or composite designs

$$
\mathcal{C D}=\left\{\theta \in L^{\infty}(\Omega ;[0,1]), A^{*}(x) \in G_{\theta(x)}, \forall x \in \Omega\right\},
$$

where, for each constant value $0 \leq \theta \leq 1, G_{\theta}$ is the set of all homogenized Hooke's law obtained by mixing the phases $A$ and $B$ in proportions $\theta, 1-\theta$.

The advantages of the relaxed formulation (2) are numerous and well described in e.g. [ALL 93], [KOH 86], [LUR 86], [MUR 85]. In particular, it always admits an optimal solution while any composite design is attained as the limit of a sequence of classical designs. This implies that relaxation does not change the problem but makes it well-posed, and that a nearly optimal classical design can easily be recovered from an optimal composite design by a suitable penalization process. There are also many numerical algorithms based on this approach that can be viewed as topology optimization methods (see e.g. [ALL 97], [ALL 93], [BEN 95], [BEN 88], [DIA 92a], [DIA 92b]).

However, in general, the relaxed formulation (2) cannot be used directly since the set $G_{\theta}$ of all composite materials is unknown. In a few special cases (of great practical importance), the optimality conditions allows to replace $G_{\theta}$ by its explicit subset of so-called sequential laminates. This is possible if the objective function $J$ and $J^{*}$ is the compliance or the first eigenfrequency (or even a sum of several of them, see e.g. [ALL 96], [ALL 00b]). In such a case, (2) is truly useful and fully explicit. Unfortunately, in all other cases, this relaxed formulation is useless since we have no knowledge of this set $G_{\theta}$ of composite materials. By opposition to what follows, we shall call (2) a fully relaxed formulation.

\section{Partial relaxation}

To obtain a tractable formulation, we restrict $G_{\theta}$ to its explicit subset $L_{\theta}$ of all sequential laminates, of any order, obtained by laminations of $A$ and $B$ in proportions $\theta$ and $(1-\theta)$. For a (given) number $q$ of laminations and unit lamination directions $\left(e_{i}\right)_{1 \leq i \leq q}$, as well as lamination parameters $\left(m_{i}\right)_{1 \leq i \leq q}$ satisfying $m_{i} \geq 0$ and $\sum_{i=1}^{q} m_{i}=1$, the Hooke's law $A^{*}$ associated to the sequential laminate with core $A$ and matrix $B$ is given by:

$$
(1-\theta)\left(A-A^{*}\right)^{-1}=(A-B)^{-1}-\theta \sum_{i=1}^{q} m_{i} f_{A}\left(e_{i}\right),
$$

with $\forall e \in \mathbb{R}^{N},|e|=1, \forall \xi$ symmetric matrix

$$
f_{A}(e) \xi: \xi=\frac{1}{\mu_{A}}\left(|\xi e|^{2}-(\xi e \cdot e)^{2}\right)+\frac{1}{\lambda_{A}+2 \mu_{A}}(\xi e \cdot e)^{2} .
$$

We introduce a set $\mathcal{L D}$ of sequentially laminated designs, defined by:

$$
\mathcal{L D}=\left\{\theta \in L^{\infty}(\Omega ;[0,1]), A^{*}(x) \in L_{\theta(x)} \forall x \in \Omega\right\} .
$$


The partial relaxation is:

$$
\inf _{\left(\theta, A^{*}\right) \in \mathcal{L D}} J^{*}\left(\theta, A^{*}\right)
$$

with the same objective function $J^{*}$ defined by (3). A priori, the existence of a minimizer of the partial relaxation (9) is not guaranteed, which is the main difference with the full relaxation (2). It seems that we have gained very little in replacing the ill-posed problem (1) by another ill-posed problem (9). Nevertheless, loosely speaking the latter is less ill-posed than the former since its integrand has been smoothed or averaged, at least partially, leading to better convexity properties. The question of how much qualitatively the partial relaxation improves on the original formulation is linked to the question of how far from optimal are the microstructures in $L_{\theta}$. As a possible justification of this partial relaxation (9), let us simply recall that in the cases of compliance or eigenfrequency optimization it coincides with the full relaxation.

For all the other cases, there is another practical advantage in using $\mathcal{L D}$ as the space of generalized designs: composites of $\mathcal{L D}$ are described by a small number of parameters. Finding optimality conditions amounts to compute the derivatives of the objective function with respect to these parameters. It allows to build numerical gradient algorithms.

To describe a numerical method, we first discretize in space the design variables $\left(\theta(x), A^{*}(x)\right)$. $\theta(x)$ denotes the total proportion of material $A$ at point $x . A^{*}(x)$ is computed using the local microstructure. If the resolution of (9) is done by finite elements, the design parameters can be chosen piecewise constant. Then, for a given value of $\theta$, a composite $A^{*} \in L_{\theta}$ is described by (6). The possible lamination directions are discretized by fixing their number to $q$ and their directions to the given vectors $\left(e_{j}\right)_{1 \leq j \leq q}$. The proportions $\left(m_{i}\right)_{1 \leq i \leq q}, m_{i}$ denoting the proportion of $A$ on direction $e_{i}$, are varying with the same spatial discretization than $\theta$. They belong to the convex set defined by the constraints $m_{i} \geq 0$ and $\sum_{i=1}^{q} m_{i}=1$. Now $J^{*}(\theta, m)$ designs the objective function depending on the discrete parameters $\theta$ and $m=\left(m_{i}\right)_{1 \leq i \leq q}$.

Partial derivatives of $J^{*}$ can be computed, introducing the adjoint state $p$ as the solution of:

$$
\left\{\begin{aligned}
-\operatorname{div}\left(A^{*} e(p)\right) & =c_{\alpha} \theta(x) C(x)\left|u(x)-u_{0}(x)\right|^{\alpha-2}\left(u(x)-u_{0}(x)\right) & & \text { in } \Omega \\
p & =0 & & \text { on } \partial \Omega
\end{aligned}\right.
$$

where $c_{\alpha}=\left(\int_{\Omega} \theta(x) C(x)\left|u(x)-u_{0}(x)\right|^{\alpha} d x\right)^{\frac{1-\alpha}{\alpha}}$.

The objective function $J^{*}(\theta, m)$ is differentiable, and if $\delta \theta(x)$ and $\delta m_{i}(x)$ are admissible increments, its directional derivative is:

$$
\delta J^{*}(\theta, m)=\int_{\Omega} \nabla_{\theta} J^{*} \delta \theta d x+\sum_{i=1}^{q} \int_{\Omega} \nabla_{m_{i}} J^{*} \delta m_{i} d x
$$


with partial derivatives

$$
\begin{aligned}
\nabla_{\theta} J^{*}(x) & =\frac{c_{\alpha}}{\alpha} C(x)\left|u(x)-u_{0}(x)\right|^{\alpha}+\ell+\frac{\partial A^{*}}{\partial \theta} e(u): e(p) \\
\nabla_{m_{i}} J^{*}(x) & =\frac{\partial A^{*}}{\partial m_{i}}(x) e(u): e(p)
\end{aligned}
$$

and

$$
\begin{aligned}
\frac{\partial A^{*}}{\partial \theta}(x) & =T^{-1}\left((A-B)^{-1}-\sum_{i=1}^{q} m_{i} f_{A}\left(e_{i}\right)\right) T^{-1} \\
\frac{\partial A^{*}}{\partial m_{i}}(x) & =-\theta(1-\theta) T^{-1} f_{A}\left(e_{i}\right) T^{-1} \\
T & =(A-B)^{-1}-\theta \sum_{i=1}^{q} m_{i} f_{A}\left(e_{i}\right) .
\end{aligned}
$$

This gives the basis for a numerical gradient method which is described in the next section. Of course, since $\theta, m_{i}$ are constrained locally at each point $x$ ( $\theta$ must stay in the range $[0,1]$, and $\left.\sum_{i=1}^{q} m_{i}=1, m_{i} \geq 0\right)$ the gradient method must be combined with a projection step to satisfy these constraints.

For simplicity we focus on the case of a single load optimization problem. There is obviously no difficulty in extending the previous analysis to multiple loads problems.

\section{Numerical algorithm}

We propose a simple gradient algorithm, with a projection step. The descent step is variable but non-optimal, which is more adapted to our applications than a real, more expensive, line-search or conjugate gradient.

1) Initialization of the design parameters $\theta_{0}, m_{i, 0}$ (for example, we take them constant satisfying the constraints).

2) Iteration until convergence, for $k \geq 0$ :

a) Computation of the state $u_{k}$ and the adjoint state $p_{k}$, solutions of (4) and (10) respectively, with the previous design parameters $\theta_{k}, m_{i, k}$.

b) Updating of these parameters by

$$
\begin{aligned}
& \theta_{k+1}=\max \left(0, \min \left(1, \theta_{k}-t_{k} \nabla_{\theta} J_{k}^{*}\right)\right) \\
& m_{i, k+1}=\max \left(0, m_{i, k}-t_{k} \nabla_{m_{i}} J_{k}^{*}+\ell_{k}\right),
\end{aligned}
$$

where $\ell_{k}$ is a Lagrange multiplier for the constraint $\sum_{i=1}^{q} m_{i, k}=1$, iteratively adjusted, and $t_{k}>0$ is a descent step such that $J^{*}\left(\theta_{k+1}, m_{k+1}\right)<J^{*}\left(\theta_{k}, m_{k}\right)$.

Remark 1: an optimal descent step $t_{k}$ can be computed by a line-search algorithm, but it could become very costly since each evaluation require the computation by finite elements of the direct elasticity problem and the adjoint state. In practice, with our 
simple method, only a few evaluations are needed for each iteration: a first step $t_{0}$ is guessed. Then, if $J_{k+1}^{*} \leq J_{k}^{*}$, the step is multiplied by a factor $>1$ for the following iterations (only one evaluation is done in this case, which is the most frequent one); if $J_{k+1}^{*}>J_{k}^{*}, t_{k}$ is divided by a factor $>1$ and $J_{k+1}^{*}$ is computed again, until it becomes smaller than $J_{k}^{*}$.

Remark 2: the gradients $\nabla_{\theta} J_{k}^{*}$ and $\nabla_{m_{i}} J_{k}^{*}$ are projected to satisfy the constraints $0 \leq \theta(x) \leq 1, \sum_{i=1}^{q} m_{i}=1$ and $m_{i} \geq 0$. In the context of shape optimization for mechanisms, where the objective function is given by (1) with $u_{0} \neq 0$, there is no link between the "performance" of the structure and its weight. It is thus possible to take $\ell=0$ in (1), removing any volume constraint on phase $A$. Otherwise, $\nabla_{\theta} J_{k}^{*}$ can be projected so that $\int_{\Omega} \theta(x) d x$ remains constant through the iterations, preserving the initial total volume fraction of $A$.

Remark 3: most of the computation time is spent in solving the successive linear elasticity problems by finite elements. If the linear systems are solved by a direct method like Crout factorization, the additional cost due to the computation of the adjoint state is small since the matrix is the same than the one involved in the elasticity problem (and it is already factorized). It is sufficient to calculate a new right-hand side and to solve the system with the previous rigidity matrix.

In $2 \mathrm{~d}$ problems, a good compromise between precision and computation time is $q=12$. An alternative could be to take less directions and add a global rotation different at each point - to the microstructure. This approach has been proposed in [ALL 00a] but it leads to stability problems due to the possible permutation in the roles of $m_{i}$ parameters when a too large rotation occurs. In $3 \mathrm{~d}$, good results are obtained with $q=91$ directions, discretizing as uniformly as possible the half unit sphere.

This algorithm gives generalized solutions with large regions made of composite material. In practice, it is a default if one wants to find classical shapes, but it is a good sign for the validity of the partial relaxation as an approximation of the true relaxation. To recover classical designs - i.e. with pure material and void - a penalization procedure is applied, as in [ALL 97], [BEN 95], [ROZ 95], forcing the density $\theta$ to prefer values 0 or 1: after convergence of the iterative process, a few additional iterations of the above algorithm, replacing (6) by a penalized formula:

$$
\left(1-\theta^{n}\right)\left(A-A^{*}\right)^{-1}=(A-B)^{-1}-\theta^{n} \sum_{i=1}^{q} m_{i} f_{A}\left(e_{i}\right)
$$

where typically $n \in[3,5]$. The fictitious material given by (12) is weaker than the original composite if $0<\theta<1$ (because $\theta^{n}<\theta$ ). Therefore, it is not advantageous to use any such composite of intermediate density. Using the modified formula (12) results in a very effective penalization scheme: almost all grey areas in the homogenized design disappear to yield a black and white "penalized" design as can be seen in the following numerical results. 


\section{REEF - 11/2002. Giens'01}

\section{Numerical results}

We propose several examples obtained with this numerical algorithm, illustrating its capabilities. In the first and the second one, the computation domain is a square with clamping conditions on the two left corners. $C(x)$ is defined as specified on figure $1, \alpha=2$ and $u_{0}(x)=(-U, 0)$ where $U>0$ is large enough, so that minimizing the objective function (3), the horizontal component of the displacement, measured on the small region where $C(x) \neq 0$, is maximized in the direction $x<0$. A force is applied in the middle of the opposite edge. According to the direction of this force, we obtain a force inverter (fig. 1) - giving a displacement opposed to the force's direction - or a Displacement multiplier (fig. 2) - which amplifies the displacement in the direction of the force.

In these examples, as well as in the 3 that follow, a small term is added to the objective function to prevent the algorithm from finding solutions where the part of the boundary supporting the loads is disconnected from the rest of the structure. Typically, on a small zone surrounding the forces' application points, $C(x)=0.1$ and $u_{0}=0$, which has to be compared to $C(x)=1$ on the region where the target displacement is wanted.

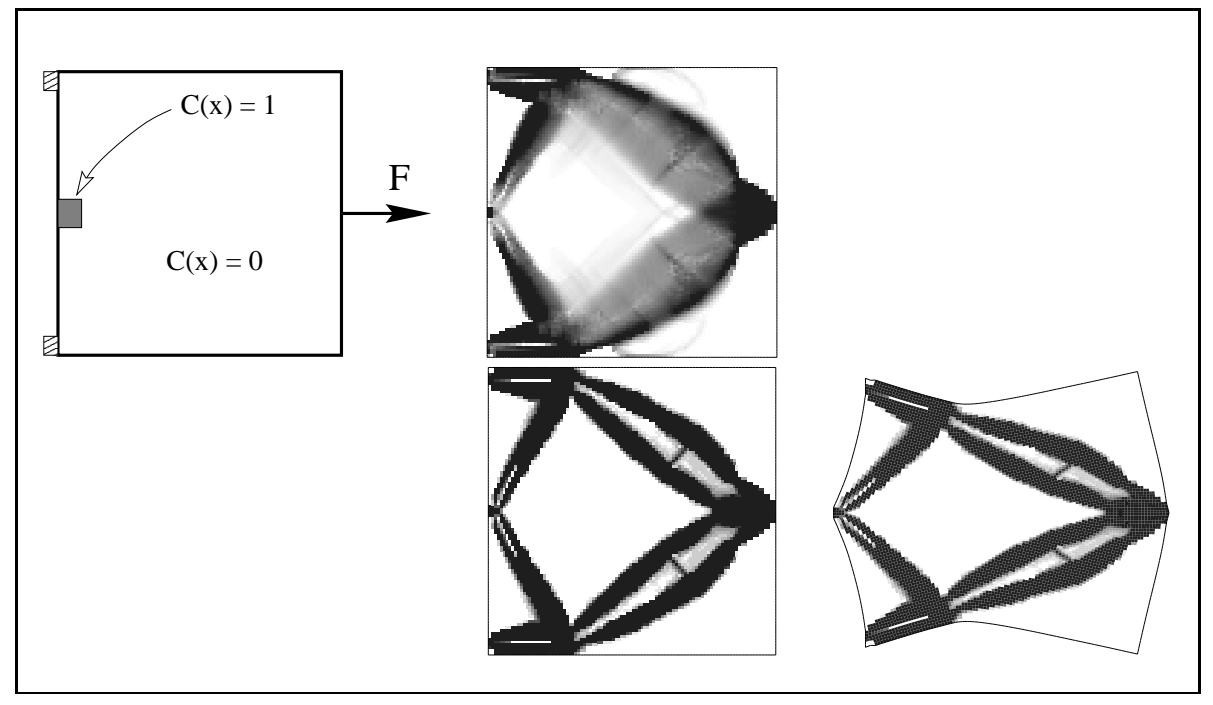

Figure 1. Force inverter. Top: setting of the problem and composite solution; bottom: penalized solution and deformed structure

In the third example (fig. 3), we want to obtain the global effect of a negative Poisson's ratio, adding reinforcing structures in a given rectangular frame. A possible application of such a device is an efficient embedding mechanism.

In the last results (fig. 4 and 5), the domain is clamped on the top and bottom faces and we want the jaws of the grip to clamp when a pressure load is applied on 
the opposite edge. Figure 5 shows a tridimensional version of the problem shown on figure 4. Remark the slight different topologies of both solutions.

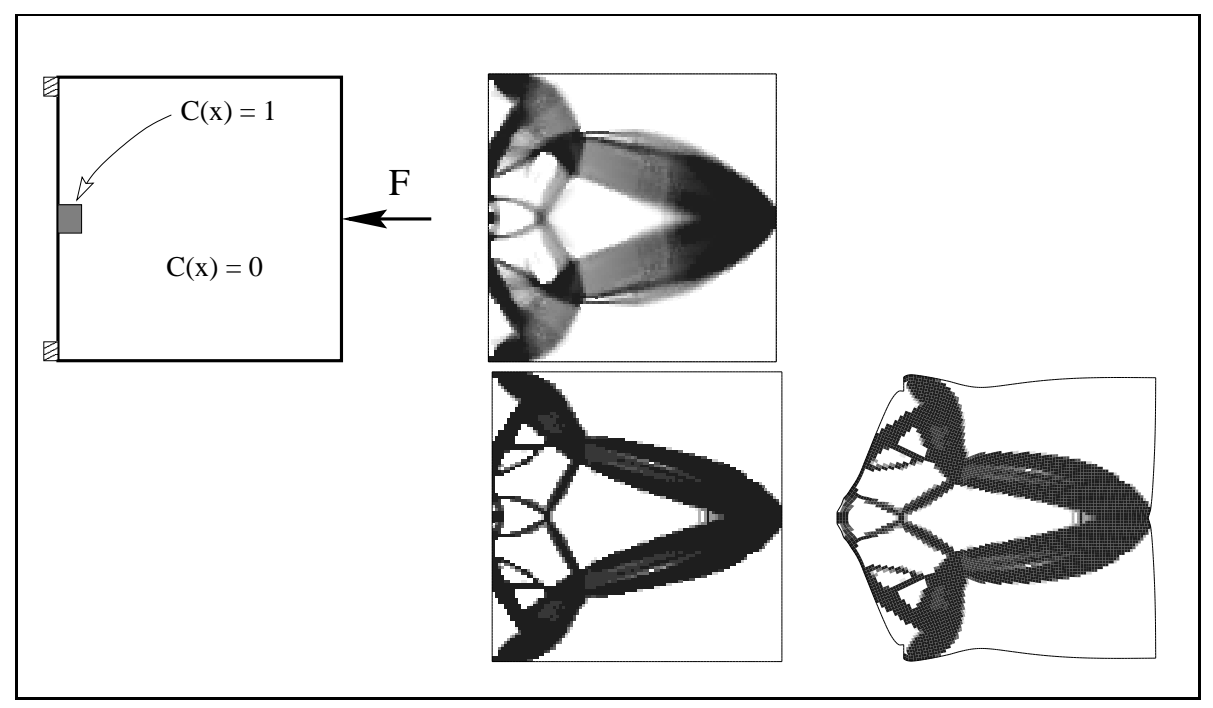

Figure 2. Displacement multiplier. Top: setting of the problem and composite solution; bottom: penalized solution and deformed structure

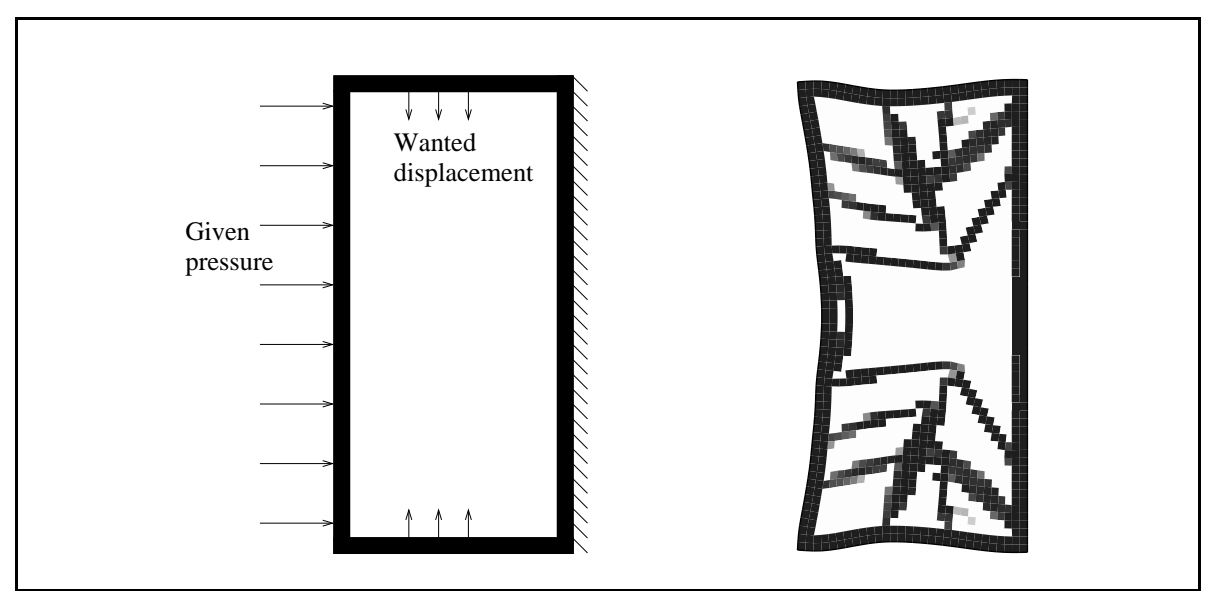

Figure 3. Mechanism with negative Poisson's ratio: setting of the problem and deformed optimal structure 


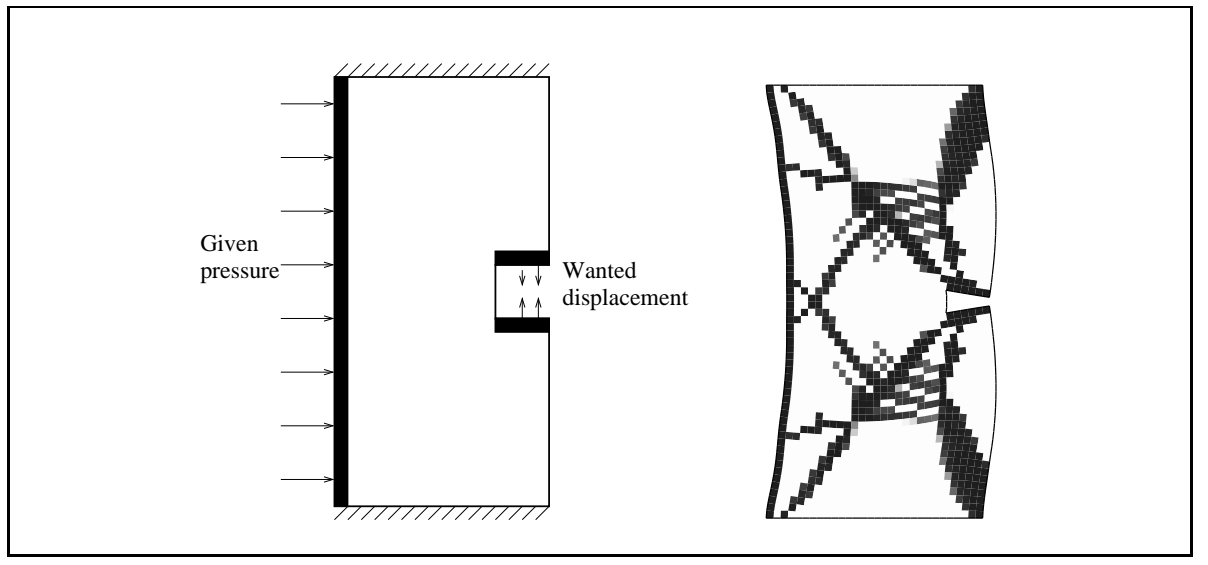

Figure 4. Grip: setting of the problem and deformed optimal structure

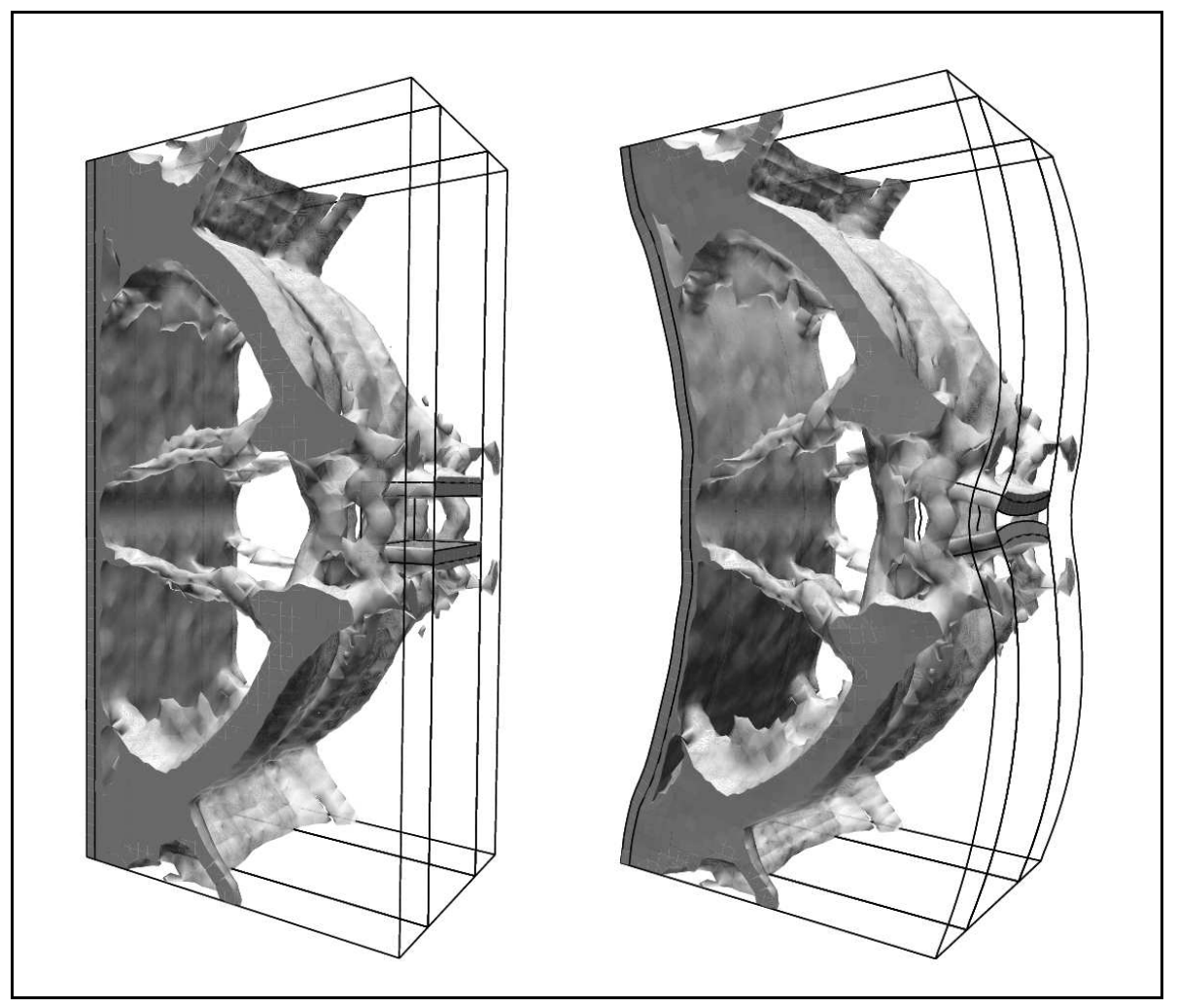

Figure 5. 3d grip: optimal structure and deformed configuration 


\section{References}

[ALL 01] Allaire G., Shape optimization by the homogenization method, Springer Verlag, New York 2001.

[ALl 00a] Allaire G., Aubry S., Jouve F., 'Shape optimization with general objective functions using partial relaxation", Topology optimization of structures and composite continua, G.I.N. Rozvany and N. Olhoffeds., p. 239-249, Kluwer Academic Publishers, 2000.

[ALl 00b] Allaire G., Aubry S., Jouve F., 'Eigenfrequency optimization in optimal design”, Comput. Methods Appl. Mech. Engrg., vol. 190, 2000, p. 3565-3579.

[All 97] Allaire G., Bonnetier E., Francfort G., Jouve F., 'Shape optimization by the homogenization method", Nümerische Mathematik, vol. 76, 1997, p. 27-68.

[ALL 96] Allaire G., Belhachmi Z., Jouve F., "The homogenization method for topology and shape optimization. Single and multiple loads case", Revue Européenne des Éléments Finis, vol. 5, 1996, p. 649-672.

[ALL 93] Allaire G., KoHn R.V., 'Optimal design for minimum weight and compliance in plane stress using extremal microstructures", Europ. J. Mech. A/Solids, vol. 12, num. 6, 1993, p. 839-878.

[BEN 95] BENDSOE M., Methods for optimization of structural topology, shape and material, Springer Verlag, New York, 1995.

[BEN 88] Bendsoe M., KIKUCHI N., 'Generating Optimal Topologies in Structural Design Using a Homogenization Method", Comp. Meth. Appl. Mech. Eng., vol. 71, 1988, p. 197224.

[CHE 97] CherKaeV A., Kohn R.V., Editors, "Topics in the mathematical modeling of composite materials", Progress in Nonlinear Differential Equations and their Applications, vol. 31, Birkhaüser, Boston, 1997.

[DIA 92a] Diaz A., BEndsoe M., 'Shape optimization of structures for multiple loading conditions using a homogenization method", Struct. Optim., vol. 4, 1992, p. 17-22.

[DIA 92b] DiAZ A., KIKUCHI N., 'Solutions to shape and topology eigenvalue optimization problems using a homogenization method", Int. J. Num. Meth. Engng., vol. 35, 1992, p. $1487-1502$

[JON 99] Jonsmann J., Sigmund O., Bouwstra S., 'Compliant thermal microactuators”, Sensors and actuators, vol. 76, 1999, p. 463-469.

[KOH 86] Kohn R.V., STRAng G., 'Optimal Design and Relaxation of Variational Problems I-II-III", Comm. Pure Appl. Math., vol. 39, 1986, p. 113-137, 139-182, 353-377.

[LUR 86] LURIE K., CHERKAEv A., 'Effective characteristics of composite materials and the optimal design of structural elements", Uspekhi Mekhaniki, vol. 9, 1986, p. 3-81. English translation in [CHE 97].

[MiN 98] Minotti P., Bourbon G., Langlet P., Masuzawa T., "Arrayed electrostatic scratch drive actuators: towards visible effects up to the human scale", J. of Intelligent Material Systems and Structures, vol. 9, 1998, p. 837-846.

[MUR 85] Murat F., Tartar L., "Calcul des Variations et Homogénéisation", in D. Bergman et. al. (eds.), Les Méthodes de l'Homogénéisation Théorie et Applications en Physique, Coll. Dir. Etudes et Recherches EDF, vol. 57, 1985, Eyrolles, Paris, p. 319369. English translation in [CHE 97]. 


\section{REEF - 11/2002. Giens'01}

[ROZ 95] Rozvany G., Bendsoe M., KIRsCh U., 'Layout optimization of structures", Appl. Mech. Reviews, vol. 48, 1995, p. 41-118.

[SIG 97] SIGMUnd O., "On the design of compliant mechanisms using topology optimization", Mech. Struct. Mach., vol. 25, 1997, p. 493-524.

[SIG 99] SIGMUND O., 'Design of multiphysics actuators using topology optimization, part I: one-material structures", DCAMM Report 632, Technical University of Denmark, 2000 